\title{
Severe Rashes Associated With Immunotherapy and Gene Mutations in a Patient With Metastatic Pancreatic Cancer
}

\section{Yanghui Ye}

Zhejiang Chinese Medical University

\section{Song Zheng ( $\nabla$ tztree@126.com )}

Hangzhou First People's Hospital

\section{Case Report}

Keywords: pancreatic cancer, sintilimab, immunotherapy, rash, SMAD4, TSC2

Posted Date: February 22nd, 2022

DOI: https://doi.org/10.21203/rs.3.rs-1374816/v1

License: (c) (i) This work is licensed under a Creative Commons Attribution 4.0 International License.

Read Full License 


\section{Abstract}

Background: Pancreatic cancer is a high-risk malignant tumor, and is traditionally treated with chemotherapy. However, the curative effect of traditional treatment is very poor, and new treatment methods are being explored. Sintilimab is a fully human IgG4 monoclonal antibody; it can bind to PD-1, block the interaction of PD-1 with its ligands, and can improve the survival of cancer patients.

Case presentation: A male metastatic pancreatic cancer patient with SMAD4 and TSC2 mutations, who had undergone three lines of systemic chemotherapy, developed severe rashes after 12 cycles of taking sintilimab combined with S-1. Considering the relationship between sintilimab and the rashes, he discontinued taking the drug, and his condition improved. Although sintilimab has shown good safety and manageable toxicity in immunotherapy, the patient in the present case developed severe rashes after 12 cycles of taking sintilimab combined with S-1.

Conclusions: Although few people have reported a severe rash caused by sintilimab, patients with SMAD4 and TSC2 mutations may more likely to develop severe rashes. We hope the case attracts the attention of oncologists.

\section{Background}

Compared with other malignant tumors, pancreatic cancer has a very poor prognosis due to its high degree of malignancy, lack of typical symptoms, and high recurrence rate. Pancreatic cancer has a high incidence and mortality, and its special structure can protect pancreatic cancer cells from chemotherapeutic agents $[1,2]$. The average five-year survival rate is less than $10 \%$ and drops to less than $3 \%$ in patients with locally advanced or metastatic pancreatic cancer [3]. After failure of second-line standard treatment, effective treatments of pancreatic cancers are limited, and some patients have a strong desire for survival.

Immune checkpoints (ICs) are important immune regulators in maintaining immune homeostasis and preventing autoimmune diseases. And blockade of the immune checkpoint pathway using immune checkpoint inhibitors (ICls) is a weapon in cancer therapy. Among all ICls, the most studied ones are antiprogrammed cell death protein 1(PD-1)/programmed cell-death protein ligand 1(PD-L1) antibodies, and anti-CTLA-4 [4]. The sintilimab is a fully human IgG4 monoclonal antibody; it can bind to PD-1, block the interaction of PD-1 with its ligands, and help recover the anti-tumor response of T-cells, and it has been approved to treat relapsed or refractory classical Hodgkin lymphoma, advanced non-small cell lung cancer (NSCLC) and metastatic hepatocellular carcinoma. Although the sintilimab has a weak immunogenicity as reflected by low detection rates of anti-drug antibodies, the toxicity was tolerable, it is associated with some unrecognized adverse reactions.

\section{Case Presentation}


A 56-year-old Chinese man who was hospitalized for six months with abdominal pain. He had no family history of cancer or drug allergies. In September 2019, magnetic resonance imaging (MRI) of the upper abdomen identified a pancreatic head mass, multiple retroperitoneal enlarged lymph nodes, and abnormal enhancement near the inferior vena cava in right lobe of the liver. Ultrasound guided biopsy of the pancreas revealed that the pathology diagnosis was pancreatic ductal adenocarcinoma (stage IV). Next generation sequencing (NGS) confirmed the tumor was microsatellite stability (MSS), and a total of 6 gene mutations, TSC2, CREBBP, HIST1H3I, MAP2K4, SMAD4, and STK11 were detected. The NGS confirmed that the tumor mutational burden (TMB) was 7.1 mut/Mb.

From October 14, 2019, the AG (gemcitabine and nab-paclitaxel) was administered for 6 courses. In April 2020, positron emission tomography/ computed tomography (PET/CT) revealed a $6.0 \mathrm{~cm} \times 6.0 \mathrm{~cm}$ mass in pancreatic head, multiple enlarged lymph nodes in different sizes were found in pancreatic head and retroperitoneum, and multiple low-density shadows in the liver. When disease progressed, he underwent endoscopic retrograde cholangiopancreatography (ERCP) and endoscopic metal biliary endoprosthesis (EMBE) due to obstructive jaundice, and changed the chemotherapy regimen to FOLFIRINOX (oxaliplatin, irinotecan, calcium folinate and 5-Fluorouracil). Before he received FOLFIRINOX, the patient had undergone 1 cycle of S-1. From April 2, 2020, he received one cycle of S-1, 8 cycles of FOLFIRINOX. The pancreatic cancer was responded to chemotherapy initially, but the disease was still progressed at later time. In October 2020, his abdomen enhanced CT scan revealed multiple liver metastases, and the largest measured $2.3 \mathrm{~cm}$. At this time, we changed the chemotherapy regimen to AG again. Before he started AG, he ate one cycle of Olaparib by himself, although he did not have the targeted drug-related gene mutations. After one cycle of AG, the disease was still progressed. In November 2020, the abdomen enhanced CT scan revealed that the size of the pancreatic head lesion was $6.4 \mathrm{~cm}$ (FIGURE 1A).

Considering the patient has undergone multi-line chemotherapy, chemotherapy alone could not inhibit the progression of the disease, and there was no standard treatment after two or more lines of systemic chemotherapy, our team decided to use chemotherapy (S-1) combined with immunotherapy (sintilimab). Sintilimab was administered intravenously ( $200 \mathrm{mg}$, q3w); and S- 1 was orally (40mg, bid, d1-14, q3w). The patient started to receive S-1 on November 24, 2020, and sintilimab on November 27, 2020. After 10 cycles, the pancreatic lesion was reduced significantly, and nearly disappeared. In June 2021, the CT scan showed that the size of the pancreatic head lesion was $1 \mathrm{~cm}$ (FIGURE 1B), decreased more than 80\% (Response Evaluation Criteria in Solid Tumors (RECIST) criteria, version 1.1).

After 12 cycles, the patient developed severe rashes (Common Terminology Criteria for Adverse Events grade 3 ) after receiving sintilimab for approximately 8 months. The dark red maculopapular rashes are frequently found on the limbs, dense below the knee, sporadic on the abdomen and buttocks, which accounts for $37 \%$ of the total body surface area (FIGURE 2A). The rash is round and dark red in color. The surface of the rash is dry without obvious bleeding, exudation and scab. The rash can cause severe pain and itching, especially in both lower limbs. The pain is graded 7-8 (numerical rating scale, NRS). Blood routine and coagulation function were basically normal. The patient was diagnosed as exudative erythema multiforme by dermatologist. He discontinued taking sintilimab due to severe rashes, and 
treated with methylprednisolone for 16 days, Piperacillin Sodium and Tazobactam Sodium (3.375 g, q8h) for 9 days. The dose of methylprednisolone was $40 \mathrm{mg}$ per day (from July 26, 2021 to July 30, 2021), 40 mg twice a day (from July 31, 2021 to August 2, 2021), 30 mg twice a day (from August 3, 2021 to August 4, 2021), 40 mg per day (from August 5, 2021 to August 7, 2021), 30 mg per day (from August 8 , 2021 to August 10, 2021). In addition, the patient was received the best supportive treatment, such as immunomodulatory (Thalidomide), anti-inflammatory (Compound Glycyrrhizin), antioxidant (GSH), antiallergic (Desloratadine Citrate Disodium), Furacilin Solution wet compress anti infection. The severe rashes disappeared gradually after discontinuation of sintilimab for 2 weeks.

About 1 month later, the rash was significantly better than before. The scab of the patient's limbs basically fell off without pruritus and pain (FIGURE 2B). The patient took S-1 alone after the rash recovered. Until now (November 2021), the rash has not recurred.

\section{Discussion}

Severe cutaneous adverse reactions belonged to type IV hypersensitivity reaction and are T cell-mediated immune responses with different effector cells and cytokines. Erythema multiforme (EM) is a rare, acute inflammatory disorder that affects skin, or mucous membranes, or both. EM is caused by a hypersensitivity reaction with the appearance of cytotoxic $T$ lymphocytes in the epithelium that induce apoptosis in keratinocytes, which leads to satellite cell necrosis. Severe erythema multiforme, also known as Stevens-Johnson syndrome (SJS), which causes widespread lesions that affect the skin, and SJS usually caused by drugs. For example, SJS related to anti-PD1 and anti-CTLA4 has also been reported, though it is uncommon. Immunocompromised individuals, such as patents with AIDS or malignancies or those who undergo immunosuppressive therapy, tend to be prone to SJS $[5,6]$.

The sintilimab is a fully human IgG4 monoclonal antibody; it can bind to PD-1, block the interaction of PD-1 with its ligands, and help recover the anti-tumor response of T-cells, and it has been approved to treat relapsed or refractory classical Hodgkin lymphoma, advanced non-small cell lung cancer (NSCLC) and metastatic hepatocellular carcinoma. The results of a phase III trial showed that the median progress free survival (mPFS) of patients with advanced nonsquamous NSCLC treated with sintilimab was 8.9 months, which was 3.9 months longer than that in the placebo group [7]. However, the incidence of grade 3 or higher adverse events was $61.7 \%$ in sintilimab group; and the immune-related adverse events (irAEs) affecting patients in the sintilimab group was $43.2 \%$, the most common irAEs were hypothyroidism, liver dysfunction, rash, etc [7].

In this case, the patient suffered severe rash after 12 cycles of sintilimab combined with S-1. The rash is the most common skin adverse reaction to anti-PD-1 therapy, which is usually mild to moderate (grade 12). However, severe rashes caused by sintilimab are rarely reported because they are rare.

Drug-induced SJS is generally believed to be a human leukocyte antigen (HLA) restricted T lymphocyte cytotoxicity [8]. Some drugs could bind to HLA and activate T cells, potentiating hypersensitivity $[9,10]$. Maintaining immunologic self-tolerance and control requires a special subset of CD4+ T cells, the Tregs 
$[11,12]$. Tregs regulate immune system through multiple mechanisms, including the release of inhibitory cytokines, the initiation of cytolysis, and the modulation of DC function [13]. The transforming growth factor- $\beta$ (TGF- $\beta$ ) family consists of pleiotropic cytokines with both proinflammatory and antiinflammatory effects, contributing to immune system homeostasis [14]. Conversion of CD4+ T cells into induced Tregs (iTregs) or peripheral Tregs (pTregs) is also dependent on TGF- $\beta[15,16]$. In mice, the lack of intact TGF- $\beta$ signaling results in an increased proinflammatory, Th2, and Th17 type response in the skin and gastrointestinal tract $[17,18]$. Th17s are a subtype of helper $T$ cells known to cause autoimmunity and inflammation [19]. TGF- $\beta$ can drive Th17 differentiation in the presence of other cytokines [20].

SMAD4 serves as the central mediator of the TGF- $\beta$ signaling pathway [21], and it is the only common mediator. TGF- $\beta$ can stimulate regulatory T-cells, which inhibit the function of other lymphocytes [22], such as TGF- $\beta$ inhibits CD8+ T-cell effector function through TGF- $\beta$ signaling pathway [23]. In pancreatic cancer, alterations of TGF- $\beta$ signaling occur through the mutation of the genes involved in the pathway (including SMAD4); this activity is present in $47 \%$ of pancreatic cancer patients [24]. And the loss of SMAD4 will abrogate the canonical TGF-B/SMAD4 signaling pathway [25].

In addition, the TSC2 can form a complex and functions by inhibiting mTORC1 kinase [26], which is a central regulator of cellular growth and metabolism [27]. There was a study showed that the PD-1 antibody treatment can increase CD8+ T-cell infiltration in TSC2-deficient human tumors [28]. In a mice study, complete absence of mTOR, naive T cells showed normal activation markers upon T cell receptor (TCR) stimulation, but failed to differentiate into Th1, Th2 and Th17 cells [29]. CD8+ T cells play an essential role in inducing drug hypersensitivities [30], and drug-specific CD8+T cells were found to predominantly proliferate during the acute stages of SJS [31]. In severe cutaneous adverse drug reactions, $C D 8+T$ cells were found to be the predominant population that infiltrated into the epidermis of skin lesions of SJS patients [32]. And CD8+ T cells were also dominant in T lymphocytes found in blister fluid [33]. The pathogenesis is driven by cytotoxic CD8+ T cells, natural killer (NK) cells and NKT cells, which are enriched in blister fluid of patients with acute SJS [33-36]. After activating certain drug-specific T lymphocytes, cytotoxic molecules ranging from granulysin to granzyme $B$ (GzmB) and a variety of proinflammatory cytokines [32, 37-39]. Besides, activated NK cells, T helper cells (Th), and APCs also contribute to the release of IL-5, IL-6, IL-12, IL-15, IL-17, IL-18, further exacerbating the extensive collateral damages and result in SJS [40,41]. For example, IL-15 can induce the production of pathogenic TNF-a, granulysin and GzmB [42], and antagonize against IL-15 has been found to inhibit delayed-type hypersensitivity (DTH) [43].

Immune checkpoint inhibitors, though quite successful in treating various cancers, have autoinflammatory side effects termed immune-related adverse events (irAEs), including the commonly seen cutaneous toxicities [44]. PD-1 is known to regulate T cell effector functions during various physiological responses, including acute and chronic infection, cancer, autoimmunity, and in immune homeostasis[45]. The proper PD-1/PD-L1 functions are important in maintaining epidermal integrity while T-cell, antibody, and cytokine responses are likely involved in irAE pathogenesis [46]. There was a study 
showed that cancer patients receiving anti-CTLA4 and/or anti-PD-1 immunotherapy suffered from more drug hypersensitivity adverse events [47]. Perivascular dermatitis, occasionally with eosinophils, may occur during PD-1 blockade but is less common [48]. In addition, blockade of the PD-1/PD-L1 pathway has further been shown to increase activation of B cells with unchecked release of anti-bodies and the production of inflammatory cytokines [49]. One case series reported that 15 of the 68 patients on anti-PD1 immunotherapy developed inflammatory skin lesions ranging from mild maculopapular rash to SJSlike lesions with expression of PD-1 on skin-infiltrating T cells and keratinocytes by immunohistochemistry [49]. What is more, the advent events can have a delayed onset, as most incidents manifest weeks to months after treatment initiation [50-53].

\section{Conclusion}

Immunotherapy faces the challenge of dealing with a double-edged sword. immunotherapy brings not only clinical benefits, but also some adverse reactions. What is more, patients with SMAD4 and TSC2 mutations may more likely to induce severe rashes. How to manage immunotherapy to maximize the therapeutic effect and avoid adverse reactions as much as possible is a problem we need to explore.

\section{Abbreviations}

ICs

immune checkpoints

$\mathrm{ICls}$

immune checkpoint inhibitors

PD-1

programmed cell death protein 1

PD-L1

programmed cell-death protein ligand 1

EM

Erythema multiforme

SJS

Stevens-Johnson syndrome

HLA

human leukocyte antigen

GzmB

granzyme B

\section{Declarations}

\section{Ethics approval and consent to participate}

Not applicable. 


\section{Patient consent for publication}

Patient consent was obtained for publication.

\section{Availability of data and materials}

Not applicable.

\section{Competing interests}

Not applicable

\section{Funding}

The National Natural Science Foundation of China (81372660), Zhejiang Province Public Welfare Technology Application Research Project (2017C33200), Medical Science Research Fund of Zhejiang Province, China (2013KYA157), and Laboratory of Clinical Cancer Pharmacology and Toxicology Research of Zhejiang Province.

\section{Author contributions}

Song Zheng is the guarantor. Yanghui Ye wrote the manuscript. All authors read, provided feedback and approved the final version.

\section{Acknowledgements}

Not applicable.

\section{References}

1. Neesse, A., et al., Stromal biology and therapy in pancreatic cancer: a changing paradigm. Gut, 2015. 64(9): p. 1476-84.

2. Torphy, R.J., Y. Zhu and R.D. Schulick, Immunotherapy for pancreatic cancer: Barriers and breakthroughs. Ann Gastroenterol Surg, 2018. 2(4): p. 274-281.

3. Siegel, R.L., K.D. Miller and A. Jemal, Cancer statistics, 2016. CA Cancer J Clin, 2016. 66(1): p. 7-30.

4. He, X. and C. Xu, Immune checkpoint signaling and cancer immunotherapy. Cell Res, 2020. 30(8): p. $660-669$.

5. Schwartz, R.A., P.H. McDonough and B.W. Lee, Toxic epidermal necrolysis: Part I. Introduction, history, classification, clinical features, systemic manifestations, etiology, and immunopathogenesis. J Am 
Acad Dermatol, 2013. 69(2): p. 173.e1-13; quiz 185-6.

6. Mittmann, N., et al., Incidence of toxic epidermal necrolysis and Stevens-Johnson Syndrome in an HIV cohort: an observational, retrospective case series study. Am J Clin Dermatol, 2012. 13(1): p. 4954.

7. Yang, Y., et al., Efficacy and Safety of Sintilimab Plus Pemetrexed and Platinum as First-Line Treatment for Locally Advanced or Metastatic Nonsquamous NSCLC: a Randomized, Double-Blind, Phase 3 Study (Oncology pRogram by InnovENT anti-PD-1-11). J Thorac Oncol, 2020. 15(10): p. 1636-1646.

8. Chang, W.C., et al., SJS/TEN 2019: From science to translation. J Dermatol Sci, 2020. 98(1): p. 2-12.

9. Wei, C.Y., et al., Direct interaction between HLA-B and carbamazepine activates T cells in patients with Stevens-Johnson syndrome. J Allergy Clin Immunol, 2012. 129(6): p. 1562-9.e5.

10. Yun, J., et al., Oxypurinol directly and immediately activates the drug-specific T cells via the preferential use of HLA-B*58:01. J Immunol, 2014. 192(7): p. 2984-93.

11. Sakaguchi, S., Naturally arising CD4+ regulatory t cells for immunologic self-tolerance and negative control of immune responses. Annu Rev Immunol, 2004. 22: p. 531-62.

12. Sakaguchi, S., et al., Regulatory T cells and immune tolerance. Cell, 2008. 133(5): p. 775-87.

13. Vignali, D.A., L.W. Collison and C.J. Workman, How regulatory T cells work. Nat Rev Immunol, 2008. 8(7): p. 523-32.

14. Weissler, K.A. and P.A. Frischmeyer-Guerrerio, Genetic evidence for the role of transforming growth factor-beta in atopic phenotypes. Curr Opin Immunol, 2019. 60: p. 54-62.

15. Chen, W., et al., Conversion of peripheral CD4+CD25- naive T cells to CD4+CD25+ regulatory $T$ cells by TGF-beta induction of transcription factor Foxp3. J Exp Med, 2003. 198(12): p. 1875-86.

16. Kanamori, M., et al., Induced Regulatory T Cells: Their Development, Stability, and Applications. Trends Immunol, 2016. 37(11): p. 803-811.

17. Anthoni, M., et al., Transforming growth factor-beta/Smad3 signalling regulates inflammatory responses in a murine model of contact hypersensitivity. Br J Dermatol, 2008. 159(3): p. 546-54.

18. Konkel, J.E., et al., Transforming Growth Factor-beta Signaling in Regulatory T Cells Controls T Helper-17 Cells and Tissue-Specific Immune Responses. Immunity, 2017. 46(4): p. 660-674.

19. Lee, G.R., The Balance of Th17 versus Treg Cells in Autoimmunity. Int J Mol Sci, 2018. 19(3).

20. Yamazaki, T., et al., CCR6 regulates the migration of inflammatory and regulatory T cells. J Immunol, 2008. 181(12): p. 8391-401.

21. Massague, J., How cells read TGF-beta signals. Nat Rev Mol Cell Biol, 2000. 1(3): p. 169-78.

22. Dardare, J., et al., SMAD4 and the TGFbeta Pathway in Patients with Pancreatic Ductal Adenocarcinoma. Int J Mol Sci, 2020. 21(10).

23. Thomas, D.A. and J. Massague, TGF-beta directly targets cytotoxic T cell functions during tumor evasion of immune surveillance. Cancer Cell, 2005. 8(5): p. 369-80. 
24. Bailey, P., et al., Genomic analyses identify molecular subtypes of pancreatic cancer. Nature, 2016. 531(7592): p. 47-52.

25. Derynck, R., et al., Human transforming growth factor-beta complementary DNA sequence and expression in normal and transformed cells. Nature, 1985. 316(6030): p. 701-5.

26. Cingarlini, S., et al., Profiling mTOR pathway in neuroendocrine tumors. Target Oncol, 2012. 7(3): p. 183-8.

27. Saxton, R.A. and D.M. Sabatini, mTOR Signaling in Growth, Metabolism, and Disease. Cell, 2017. 168(6): p. 960-976.

28. Liu, H.J., et al., TSC2-deficient tumors have evidence of T cell exhaustion and respond to anti-PD1/anti-CTLA-4 immunotherapy. JCl Insight, 2018. 3(8).

29. Delgoffe, G.M., et al., The mTOR kinase differentially regulates effector and regulatory $T$ cell lineage commitment. Immunity, 2009. 30(6): p. 832-44.

30. Rozieres, A., et al., CD8+ T cells mediate skin allergy to amoxicillin in a mouse model. Allergy, 2010. 65(8): p. 996-1003.

31. Hanafusa, $T$., et al., The predominant drug-specific T-cell population may switch from cytotoxic $T$ cells to regulatory $T$ cells during the course of anticonvulsant-induced hypersensitivity. J Dermatol Sci, 2012. 65(3): p. 213-9.

32. Chung, W.H., et al., Granulysin is a key mediator for disseminated keratinocyte death in StevensJohnson syndrome and toxic epidermal necrolysis. Nat Med, 2008. 14(12): p. 1343-50.

33. Nassif, A., et al., Toxic epidermal necrolysis: effector cells are drug-specific cytotoxic T cells. J Allergy Clin Immunol, 2004. 114(5): p. 1209-15.

34. Le Cleach, L., et al., Blister fluid T lymphocytes during toxic epidermal necrolysis are functional cytotoxic cells which express human natural killer (NK) inhibitory receptors. Clin Exp Immunol, 2000. 119(1): p. 225-30.

35. Leyva, L., et al., Anticonvulsant-induced toxic epidermal necrolysis: monitoring the immunologic response. J Allergy Clin Immunol, 2000. 105(1 Pt 1): p. 157-65.

36. Nassif, A., et al., Drug specific cytotoxic T-cells in the skin lesions of a patient with toxic epidermal necrolysis. J Invest Dermatol, 2002. 118(4): p. 728-33.

37. Posadas, S.J., et al., Delayed reactions to drugs show levels of perforin, granzyme B, and Fas-L to be related to disease severity. J Allergy Clin Immunol, 2002. 109(1): p. 155-61.

38. Caproni, M., et al., Expression of cytokines and chemokine receptors in the cutaneous lesions of erythema multiforme and Stevens-Johnson syndrome/toxic epidermal necrolysis. Br J Dermatol, 2006. 155(4): p. 722-8.

39. Abe, R., et al., Granulysin as a marker for early diagnosis of the Stevens-Johnson syndrome. Ann Intern Med, 2009. 151(7): p. 514-5.

40. Chen, C.B., et al., An Updated Review of the Molecular Mechanisms in Drug Hypersensitivity. J Immunol Res, 2018. 2018: p. 6431694. 
41. Hashizume, H., T. Fujiyama and Y. Tokura, Reciprocal contribution of Th17 and regulatory T cells in severe drug allergy. J Dermatol Sci, 2016. 81(2): p. 131-4.

42. Su, S.C., et al., Interleukin-15 Is Associated with Severity and Mortality in Stevens-Johnson Syndrome/Toxic Epidermal Necrolysis. J Invest Dermatol, 2017. 137(5): p. 1065-1073.

43. Kim, Y.S., et al., Targeting the IL-15 receptor with an antagonist IL-15 mutant/Fc gamma2a protein blocks delayed-type hypersensitivity. J Immunol, 1998. 160(12): p. 5742-8.

44. Sibaud, V., Dermatologic Reactions to Immune Checkpoint Inhibitors: Skin Toxicities and Immunotherapy. Am J Clin Dermatol, 2018. 19(3): p. 345-361.

45. Sharpe, A.H. and K.E. Pauken, The diverse functions of the PD1 inhibitory pathway. Nat Rev Immunol, 2018. 18(3): p. 153-167.

46. Postow, M.A., R. Sidlow and M.D. Hellmann, Immune-Related Adverse Events Associated with Immune Checkpoint Blockade. N Engl J Med, 2018. 378(2): p. 158-168.

47. Ford, M., et al., High proportion of drug hypersensitivity reactions to sulfasalazine following its use in anti-PD-1-associated inflammatory arthritis. Rheumatology (Oxford), 2018. 57(12): p. 2244-2246.

48. Kaunitz, G.J., et al., Cutaneous Eruptions in Patients Receiving Immune Checkpoint Blockade: Clinicopathologic Analysis of the Nonlichenoid Histologic Pattern. Am J Surg Pathol, 2017. 41(10): p. 1381-1389.

49. Thibult, M.L., et al., PD-1 is a novel regulator of human B-cell activation. Int Immunol, 2013. 25(2): p. $129-37$.

50. Hwang, S.J., et al., Cutaneous adverse events (AEs) of anti-programmed cell death (PD)-1 therapy in patients with metastatic melanoma: A single-institution cohort. J Am Acad Dermatol, 2016. 74(3): p. 455-61.e1.

51. Saw, S., H.Y. Lee and Q.S. Ng, Pembrolizumab-induced Stevens-Johnson syndrome in non-melanoma patients. Eur J Cancer, 2017. 81: p. 237-239.

52. Nayar, N., K. Briscoe and P.P. Fernandez, Toxic Epidermal Necrolysis-like Reaction With Severe Satellite Cell Necrosis Associated With Nivolumab in a Patient With Ipilimumab Refractory Metastatic Melanoma. J Immunother, 2016. 39(3): p. 149-52.

53. Jour, G., et al., Autoimmune dermatologic toxicities from immune checkpoint blockade with anti-PD-1 antibody therapy: a report on bullous skin eruptions. J Cutan Pathol, 2016. 43(8): p. 688-96.

\section{Figures}

\section{Figure 1}

Enhanced CT images. The pancreatic mass indicated by the arrow on upper-abdomen enhanced CT took in November 2020 (A), in June 2021 (B). After sintilimab combined with S-1 treatment, the pancreatic 
lesion was significantly reduced and nearly disappeared. The pancreatic head lesion was decreased more than $80 \%$.

\section{Figure 2}

The severe rashes after receiving sintilimab for approximately 8 months. The dark red maculopapular rashes are frequently found on the limbs, dense below the knee, sporadic on the abdomen and buttocks. The rash is round and dark red in color. The surface of the rash is dry without obvious bleeding, exudation After almost 1 month, the scab of limbs were basically fell off. 